\title{
PERSPECTIVE
}

\section{Potential synergistic implications for stromal-targeted radiopharmaceuticals in bone-metastatic prostate cancer}

\author{
Oliver Sartor
}

Genetic heterogeneity and chemotherapy-resistant 'stem cells' represent two of the most pressing issues in devising new strategies for the treatment of advanced prostate cancer. Though curative strategies have long been present for men with localized disease, metastatic prostate cancer is currently incurable. Though substantial improvements in outcomes are now possible through the utilization of newly approved therapies, novel combinations are clearly needed. Herein we describe potentially synergistic interactions between bone stromal-targeted radiopharmaceuticals and other therapies for treatment of bone-metastatic prostate cancer. Radiation has long been known to synergize with cytotoxic chemotherapies and recent data also suggest the possibility of synergy when combining radiation and immune-based strategies. Combination therapies will be required to substantially improve survival for men with castrate-resistant metastatic prostate cancer and we hypothesize that bone-targeted radiopharmaceuticals will play an important role in this process.

Asian Journal of Andrology (2011) 13, 366-368; doi:10.1038/aja.2011.23; published online 18 April 2011

Keywords: bone; immunotherapy; metastasis; prostate cancer; radiopharmaceuticals; radium; samarium; stroma; strontium; XL-184

\section{INTRODUCTION}

Bone-seeking radiopharmaceuticals such as samarium-153 lexidronam and strontium- 89 have been approved by regulatory authorities for use in the palliative treatment of bone pain associated with metastatic cancer. These agents bind to bone stromal elements by various mechanisms and radiate both adjacent cancerous lesions and their associated microenvironment. Data to date have demonstrated palliative but not survival benefits for these agents. Herein we provide a rationale for utilizing bone-targeted radiopharmaceuticals in potentially synergistic combinatorial strategies designed to alter the natural history of cancerous lesions. To understand the rationale for utilizing targeted radiopharmaceuticals in this manner, one must first understand both the heterogeneity and stem cell-like characteristics of metastatic cancer as these concepts are integral to understanding how radiopharmaceuticals fundamentally differ from conventional anticancer therapies such as chemotherapy and hormonal manipulation.

\section{GENETIC HETEROGENEITY}

Genetic studies of cancer highlight the heterogeneity of this complex disease. ${ }^{1}$ Genome-wide mutational studies in prostate cancer are yet to be reported in detail, but initial data seemingly recapitulate studies in other tumors which demonstrate considerable variation from patient to patient. A comprehensive analysis of more than 20000 genes in two tumor types found that typical solid tumors (pancreatic cancer and glioblastoma) contained about 60 distinct mutations. However, no two patients had the exact same set of mutations. ${ }^{2,3}$ One of the authors was quoted as saying that 'If you have 100 patients, you have 100 different diseases'. ${ }^{4}$ Mutational analysis captures only a small part of the total genetic heterogeneity. Chromosomal instability with copy number variation including duplications, amplifications and deletions create even more genomic chaos and such alterations are common in human solid cancers. ${ }^{1,5}$

Genetic heterogeneity represents one of the fundamental problems in cancer therapeutics today. How can clinicians use targeted therapy against tumor cells that are so genetically heterogeneous? This heterogeneity allows 'Darwinian' forces to conspire so that virtually all metastatic solid tumors have evaded cure despite decades of intensive research. ${ }^{5}$ Selective pressures (therapies) are prescribed by clinicians, but those strategies are countered by proliferative responses in resistant cancer cells derived from heterogeneous populations of cells. Such responses are a fundamental truth in current cancer therapeutics and in prostate cancer are recently exemplified by the development of ligand-independent androgen receptors in patients progressing after androgen deprivation. ${ }^{6,7}$

\section{CANCER STEM CELLS}

It is evident from a variety of model systems that not all cancer cells are pluripotent. Some cells serve a more important role in the establishment and propagation of tumors than others. Rightly or wrongly, these critical cells have been termed 'cancer stem cells'. ${ }^{8}$ Detection of circulating tumor cells is now possible in the majority of patients with metastatic prostate cancer using sensitive techniques, ${ }^{9}$ but only a very small minority of these cells will ever propagate and lead to metastatic tumors. It is probable that cancer 'stem cells' are key to establishment of metastatic tumors and that these cells are thought to be relatively undifferentiated. ${ }^{10}$ It has been proposed that that 
prostate-specific antigen-producing cells are a derivative of 'stem cells' (just as neutrophils are derivatives of stem cells in chronic myeloid leukemia). Viewed in this context, one might keep in mind the possibility that the prostate-specific antigen-producing cell prostate cancer cell is a relative bystander and not truly the source of the problem. Taken together, one is left with impression that neither endocrine therapies nor chemotherapies (even our newest and best ones) will result in cures for patients with metastatic prostate cancer.

In summary, we view the genetic heterogeneity of cancer and the stem cell that is resistant to both chemotherapy and hormonal therapies as the dual challenges that we must face and overcome in order to cure metastatic prostate cancer.

\section{ECOLOGICAL NICHES UNIQUE TO PROSTATE CANCER}

Metastatic prostate cancer is a remarkably bone-tropic disease. Data from large clinically series indicate that approximately $90 \%$ of patients with metastatic castrate-resistant prostate cancer will have radiographic evidence of bone metastases, the highest rate encountered in any solid tumor. ${ }^{11}$ Given that the bone is a preferential site of growth, it is reasonable to hypothesize that specific interactions between tumorigenic stem cells and the bone microenvironmental 'niches' are critical for tumor progression and metastasis. These environmental 'niches' are poorly understood but represent a potentially provocative strategy for novel therapeutics. Targeting these 'niches' effectively will potentially require a stromal-targeted therapy. We note that a stromal-targeted therapy may avoid some of the perplexing issues associated with tumor cell heterogeneity. By targeting the relatively homogenous stroma, some of the difficulties associated with targeting a highly genetically heterogeneous cancer cell could be obviated.

\section{RADIATION AND THE STEM CELL}

Killing a cancerous stem cell remains a challenge, particularly when they are widely distributed as is the case for most patients with metastatic disease. Reliable strategies for killing cancer stem cells are difficult to develop but one possibility has been in hand for over a century. Marie Sklodowska Curie recognized that radioactive treatments could positively impact cancer patients ${ }^{12}$ and the ability of radiation to cure localized cancers is well recognized. We know that radiation can kill a stem cell, but the effectiveness of radiation against metastatic cancer is hindered by the inability of conventional radiation techniques to effectively target disseminated disease.

Bone-seeking radiopharmaceuticals have the ability to target disseminated tumors via selective binding to bone stromal elements that are altered as a consequence of metastatic deposits. ${ }^{13}$ These agents localize their binding to the same space that prostate cancers occupy in their preferred metastatic site (bone). In Figure 1, images derived from a conventional Tc-99 MDP bone scan and an Sm-153 EDTMP scan are shown. No differences are seen in areas of bone uptake, thus demonstrating the successful localization of Sm-153 EDTMP (with its attendant beta-emission) to the regions occupied by bony metastases.

Two radiopharmaceuticals (Sr-89 and Sm-153 EDTMP) are currently approved for the treatment of metastatic bone pain in prostate cancer patients and both of these are beta-emitters. A beta particle is an electron; when used therapeutically alone in patients with metastatic castrate-resistant prostate cancer (mCRPC), isotopes have a palliative effect on pain but do not prolong survival. ${ }^{14}$

Several small but provocative studies in MCRPC suggest that combinations of a bone-seeking radiopharmaceutical and a chemotherapy may provide synergy and alter the natural history of the underlying

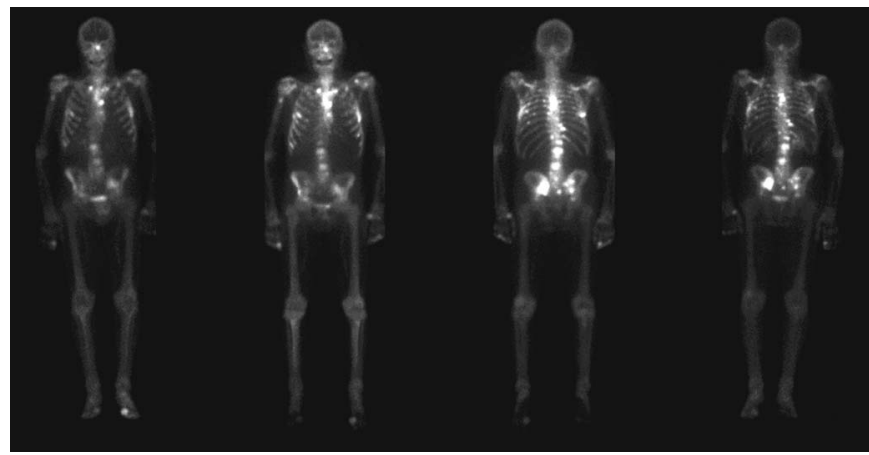

Figure 1 Comparison of bone scan images with $\mathrm{TC}^{99}-\mathrm{MDP}$ (far left and far right) and $\mathrm{Sm}^{153}$-EDTMP (middle left and middle right) (images courtesy of Dr Todd Hoover).

cancer. A randomized phase II studies conducted at MD Anderson suggested that survival may be prolonged by using a combination of a beta-emitting bone-seeking isotope in combination with doxorubicin chemotherapy. ${ }^{15}$ This trial, conducted in patients with bone-mCRPC, utilized a combination of strontium-89 and doxorubicin, and demonstrated a substantial survival advantage for patients treated with combination therapy as opposed to those patients treated with doxorubicin alone. These small randomized phase II findings have yet to be confirmed in a phase III setting. Subsequent studies with Sm-153 EDTMP and docetaxel ${ }^{16}$ in mCRPC suggested that this combination may overcome docetaxel resistance in patients previously been treated with docetaxel alone. Should this finding be confirmed in larger studies, it will provide additional support for the concept that bone-targeted radiopharmaceuticals can synergize with chemotherapy in a manner that alters the natural history of the underlying cancerous lesion.

Radium-223 is a bone stromal-targeted radiopharmaceutical with an alpha emission. The alpha particle consists of two protons and two neutrons, and is considerably more destructive to tumor cells than a beta particle. Alpha particles have been shown to induce apoptosis in human hematopoietic stem $\left(\mathrm{CD} 34^{+}\right)$stem cells. ${ }^{17}$ Radium-223 has a very high linear energy transfer and only $1-5$ hits per cell can be fatal. Double-strand breaks are induced even in quiescent cells and low oxygen levels. ${ }^{17}$ Paradoxically, because of a very short track radius post-deposition in bone stroma, the potential suppression of normal bone marrow function is minimal. In addition to hitting the tumor cell directly, it is possible that the bone stromal 'niche' in the area of tumor cell deposition is altered in a potentially favorable way by alpha-particle emission. Radiated areas of bone are known to be relatively impervious to subsequent metastatic disease, ${ }^{18}$ presumably by altering the stroma microenvironment.

A preliminary randomized small phase II trial with radium-223 administered in four doses (monthly injections) indicated a possible survival benefit in metastatic castrate-resistant prostate cancer patients. ${ }^{19}$ Side effects were not clearly distinguishable from placebo. Currently a large randomized phase III with an overall survival endpoint is underway in MCRPC patients with six doses of radium-223. This trial has recently completed accrual (January 2011) with over 900 patients enrolled.

\section{OTHER COMBINATIONS POTENTIALLY SYNERGISTIC WITH RADIOPHARMACEUTICALS}

Most in the field of oncology understand that multiple concomitant therapies will be required to cure metastatic cancer. The original 
curative studies from Hodgkin's disease and other types of lymphoma determined that at least four drugs (or radiation) are needed for cure. $^{20}$ Solid tumors such as prostate cancer are genomically complex diseases and such tumors (when metastatic) represent an enormous challenge. It is unlikely that any single therapy will suffice in curing metastatic prostate cancer.

Though the ability of radiation to kill tumor cells directly is well recognized, multiple studies also indicate that radiation may help regulate tumor antigens essential for immune recognition. ${ }^{21-23}$ Thus, there is a potential opportunity for using radiation therapy (radiopharmaceuticals and/or external beam radiation) in combination with newer immunotherapies such as sipuleucel-T, PROSTVACVF/TRICOM or ipilumimab. Such combinations are logical and potentially synergistic. Combinations of bone-targeted radiopharmaceuticals and immunotherapies need exploration sooner rather than later.

Radiation and chemotherapy combinations are well recognized to be synergistic in a variety of cancers. For diseases such as locally advanced cervical cancer, combinations of radiation and chemotherapy represent the standard of care based on studies which demonstrate improvements in overall survival. There is limited experience for combinations of radiopharmaceuticals and chemotherapy in metastatic prostate $\operatorname{cancer}^{15,16}$ (vide supra), but this limited experience is positive rather than negative. Combinations of radium-223 and docetaxel are now underway in patients with mCRPC. Eventually, triple combinations of chemotherapy, radiation/ radiopharmaceutical and immunotherapy should be tested. Clinical trials assessing these combinations exploring end points that include quality or quantity of life will be the acid test.

Some data indicate that bone-targeted radiopharmaceuticals may synergize with agents which inhibit angiogenesis. ${ }^{21}$ Recent data using a novel vascular endothelial growth factor receptor/C-met inhibitor (XL-184 or cabozantinib) indicate a strong inhibitory effect on bone metastatic disease. ${ }^{24}$ Combinations of bone-seeking radiopharmaceuticals such as radium-223 and compounds such as XL-184 may provide an opportunity for significant synergy given the distinct but complimentary mechanisms of therapeutic action. Such combinations should be evaluated sooner rather than later given a strong theoretical rationale.

\section{CONCLUSION}

MCRPC patients are challenging and all therapies to date have had modest effects. Multiple potentially synergistic combinations involving stromal-targeted therapies with radiopharmaceuticals such as radium-223 and immunotherapy and/or chemotherapy are rational in concept. Though hypotheses can be generated from animal models, proof of effectiveness will occur only in the clinic. Overcoming genetic heterogeneity and the challenge of disseminated cancer stem-like cells will not be easy, but targeting bone stromal niches with radiopharmaceuticals in combination with immunotherapies, chemotherapies and/or vascular targeted therapies might be a good place to start.

\section{COMPETING FINANCIAL INTERESTS}

Prof Oliver Sartor is the consultant to Algeta, Exelixis, SanofiAventis, Dendreon, Bellicum, Ortho-Biotech, EUSA, Millenium/ Takeda, Medivation, Oncogenex, TEVA, and Pfizer.

1 Beroukhim R, Mermel CH, Porter D, Wei G, Raychaudhuri S et al. The landscape of somatic copy-number alteration across human cancers. Nature 2010; 463: 899-905.

2 Jones S, Zhang X, Parsons DW, Lin JC, Leary RJ et al. Core signaling pathways in human pancreatic cancers revealed by global genomic analyses. Science 008321 : 1801-6.

3 Parsons DW, Jones S, Zhang X, Lin JC, Leary RJ et al. An integrated genomic analysis of human glioblastoma multiforme. Science 2008; 321: 1807-12.

4 http://www.hhmi.org/news/vogelstein20080904.html (accessed 1 November 2010).

5 Gerlinger M, Swanton C. How Darwinian models inform therapeutic failure initiated by clonal heterogeneity in cancer medicine. Br J Cancer 2010; 103: 1139-43.

6 Nacusi LP, Tindall DJ. Androgen receptor abnormalities in castration-recurrent prostate cancer. Expert Rev Endocrinol Metab 2009; 4: 417-22.

7 Guo Z, Yang X, Sun F, Jiang R, Linn DE et al. A novel androgen receptor splice variant is up-regulated during prostate cancer progression and promotes androgen depletionresistant growth. Cancer Res 2009; 69: 2305-13.

8 Taylor RA, Toivanen R, Risbridger GP. Stem cells in prostate cancer: treating the root of the problem. Endocr Relat Cancer 2010; 17: R273-85.

9 Nagrath S, Sequist LV, Maheswaran S, Bell DW, Irimia D et al. Isolation of rare circulating tumour cells in cancer patients by microchip technology. Nature 2007; 450: 1235-9.

10 Guzmán-Ramírez N, Völler M, Wetterwald A. In vitro propagation and characterization of neoplastic stem/progenitor-like cells from human prostate cancer tissue. Prostate 2009; 69: 1683-93.

11 Tannock IF, de Wit R, Berry WR, Horti J, Pluzanska A et al. TAX 327 Investigators. Docetaxel plus prednisone or mitoxantrone plus prednisone for advanced prostate cancer. N Engl J Med 2004; 351: 1502-12.

12 Laugier A. The first century of radiotherapy in France. Bull Acad Natl Med 1996; 180: 143-60.

13 Loberg RD, Logothetis CJ, Keller ET, Pienta KJ. Pathogenesis and treatment of prostate cancer bone metastases: targeting the lethal phenotype. J Clin Oncol 2005; 23: 8232-41.

14 Sartor O, Reid RH, Hoskin PJ, Quick DP, Ell PJ et al. Quadramet 424Sm10/11 Study Group. Samarium-153-Lexidronam complex for treatment of painful bone metastases in hormone-refractory prostate cancer. Urology 2004; 63: 940-5.

15 Tu SM, Millikan RE, Mengistu B, Delpassand ES, Amato RJ et al. Bone-targeted therapy for advanced androgen-independent carcinoma of the prostate: a randomised phase II trial. Lancet 2001; 357: 336-41.

16 Morris MJ, Pandit-Taskar N, Carrasquillo J, Divgi CR, Slovin S et al. Phase I study of samarium-153 lexidronam with docetaxel in castration-resistant metastatic prostate cancer. J Clin Oncol 2009; 27: 2436-42.

17 Anderson RM, Stevens DL, Sumption ND, Townsend KM, Goodhead DT et al. Effect of linear energy transfer (LET) on the complexity of alpha-particleinduced chromosome aberrations in human CD34 ${ }^{+}$cells. Radiat Res 2007; 167: $541-50$.

18 Kaplan ID, Valdagni R, Cox RS, Bagshaw MA. Reduction of spinal metastases after preemptive irradiation in prostatic cancer. Int J Rad Onc Biol Phys 1990; 18: 101925.

19 Nilsson S, Franzén L, Parker C, Tyrrell C, Blom R et al. Bone-targeted radium-223 in symptomatic, hormone-refractory prostate cancer: a randomised, multicentre, placebo-controlled phase II study. Lancet Oncol 2007; 8: 587-94.

20 de Vita VT Jr, Hubbard SM, Longo DL. Treatment of Hodgkin's disease. J Nat/ Cancer Inst Monogr 1990; 10: 19-28.

21 Kamrava M, Bernstein MB, Camphausen K, Hodge JW. Combining radiation, immunotherapy, and antiangiogenesis agents in the management of cancer: the Three Musketeers or just another quixotic combination? Mol Biosyst 2009; 5: 1262-70.

22 Ferrara TA, Hodge JW, Gulley JL. Combining radiation and immunotherapy for synergistic antitumor therapy. Curr Opin Mol Ther 2009; 11: 37-42.

23 Demaria S, Bhardwaj N, McBride WH, Formenti SC. Combining radiotherapy and immunotherapy: a revived partnership. Int J Radiat Oncol Biol Phys 2005; 63: 655-66.

24 Smith DC, Smith MR, Small EJ, Sweeney C, Kurzrock R, Gordon MR et al. Phase 2 study of XL184 in a cohort of patients with castration resistant prostate cancer (CRPC) and measurable soft tissue disease. EJC Supp/ 2011; 8: 129. 